\title{
HarassMap: Using Crowdsourced Data to Map Sexual Harassment in Egypt Chelsea Young
}

\author{
"Crowdsourcing is the process by which the power of the" \\ many can be leveraged to accomplish feats that were \\ once the province of a specialized few.
}

\author{
Jeff Howe \\ Contributing Editor, Wired Magazine \\ tinyurl.com/lxbf7
}

\begin{abstract}
Through a case study of HarassMap, an advocacy, prevention, and response tool that uses crowdsourced data to map incidents of sexual harassment in Egypt, this article examines the application of crowdsourcing technology to drive innovation in the field of social policy. This article applies a framework that explores the potential, limitations, and future applications of crowdsourcing technology in this sector to reveal how crowdsourcing technology can be applied to overcome cultural and environmental constraints that have traditionally impeded the collection of data. Many of the lessons emerging from this case study hold relevance beyond the field of social policy. Applied to specific problems, this technology can be used to improve the efficiency and effectiveness of mitigation strategies, while facilitating rapid and informed decision making based on "good enough" data. However, this case also illustrates a number of challenges arising from the integrity of crowdsourced data and the potential for ethical conflict when using this data to inform policy formulation.
\end{abstract}

\section{Introduction}

A recent report by UN Women found that $99.3 \%$ of women in Egypt report being sexually harassed and $49.2 \%$ report that this harassment occurs on a daily basis (United Nations, 2013; tinyurl.com/oxshu8h). This harassment occurs regardless of the women's age, their attire, or whether or not they elect to wear the hijab. Despite these alarming findings, victims of sexual harassment continue to face a high degree of stigma and shame. As a result, sexual harassment in Egypt goes largely unreported.

In 2010, Pennsylvania-born Rebecca Chiao decided to take a stand against the widespread sexual harassment of women on Egyptian streets, and with the help of several others, she developed HarassMap (harassmap.org), a crowdsourcing-based advocacy, prevention, and response tool that maps incidents of sexual harassment. Harassmap was inspired by the use of FrontlineSMS (frontlinesms.com) to distribute and collect information via text messages (SMS; tinyurl.com/d7mgla5), and it uses open source software that was originally developed by Ushahidi (ushahidi.com) for reporting and mapping incidents of post-election violence in Kenya.

HarassMap was originally launched as an initiative that was entirely run by volunteers. In 2012, the project received a two-year grant from Canada's International Development Research Centre (IDRC; idrc.ca) to explore the potential of crowdsourcing as a research methodology. This funding allowed HarassMap to bring on fulltime paid staff; however, the initiative continues to rely heavily on its over 1000 volunteers (HarassMap; tinyurl.com/nwsojeg). At present, in light of the legal restrictions placed on the formation of civil society organizations in Egypt, HarassMap is currently operating as an incubated project of Nahdet el Mahrousa (tinyurl.com/ k3b9tg4), an established civil society organization that provides capacity building and technical support to emerging social enterprises.

The data submitted by HarassMap users is used to support offline community mobilization campaigns that 


\section{HarassMap: Using Crowdsourced Data to Map Sexual Harassment in Egypt}

Chelsea Young

are designed to break down misconceptions surrounding sexual harassment, and it is used to challenge the social acceptability of such behaviour. By allowing victims to anonymously report sexual harassment, HarassMap hopes to overcome the cultural and institutional barriers that otherwise prevent women from reporting harassment. This innovative application of crowdsourcing technologies to address a social problem has proven successful in raising awareness and engaging community members to stop the taboo subject of sexual harassment in Egyptian society. HarassMap's success has garnered significant international attention, leading Harassmap's founders to provide coaching to activists in 28 countries and to assist in the establishment of HarassMap-inspired programs in eight of these countries (tinyurl.com/nm9ngm9).

In order to draw out some general lessons from this case, this article examines the application of this emerging technology in terms of its potential, limitations, and future applications. First, we examine the crowdsourcing technology and its potential impact at the individual, community, national, and global levels. Next, we discuss some of the limitations of this technology and their impact in the context of the HarassMap project. Then, we identify possible future applications of this technology. Finally, we conclude with insights into the potential of crowdsourcing technology to drive innovation in fields beyond social policy.

\section{Potential Benefits}

The most obvious potential benefit of HarassMap is to help individual victims and prevent others from incidents of harassment. However, further impacts may be felt at the community, national, and global levels, as discussed in the subsections that follow.

\section{Benefits to individuals}

At the level of the individual, the application of crowdsourcing technology enables HarassMap to overcome constraints that often prevent victims from reporting sexual harassment or from seeking the necessary support services to overcome trauma. This approach insulates victims from the social stigma and shame associated with sexual harassment, and it overcomes barriers in formal law enforcement channels that prevent the reporting and prosecution of these offences.

However, when considering the impact of this initiative on the individual, it is important to first discuss the nature of the problem that HarassMap seeks to address.
Sexual harassment has a direct negative impact on women's mobility and participation in the public sphere. According to the Ilahi (2009; tinyurl.com/7rxu6hf), the fear of sexual harassment has led women to adapt their public behaviour by, for example, changing their appearance, avoiding eye contact with men, travelling only with a male companion, or restricting their movement to certain areas or times of the day. Unfortunately, this situation perpetuates a social construction of women devoid of sexuality and absent from the public sphere, and it leaves women vulnerable to accusations of blame for the harassment that they do experience if they have not conformed to traditional feminine roles linked to their behaviour and attire (Ilahi, 2009; tinyurl.com/ 7rxu6hf).

HarassMap helps to overcome these challenges by providing a platform where women can easily document instances of sexual harassment. Through the use of social media or SMS technology, women can share their experiences anonymously, free from the social stigma and accusations of blame. This model helps to facilitate social protection and greater social welfare by establishing a community of victims based on shared experiences.

The crowdsourcing model also allows victims to bypass institutional constraints within formal law enforcement channels that may prevent them from reporting incidents of sexual harassment. Such constraints are acknowledged in the Arab Human Development Report (2006; tinyurl.com/m5gpqr3), where it is noted that several Arab penal codes fail to adequately criminalize sexual harassment. Even when such behaviour is formally criminalized, Ambrosetti (2013; tinyurl.com/ptdaqab) explains that there is no guarantee that such laws will be able to overcome the "hegemonic perceptions and patriarchal norms" embedded in informal institutions to allow for their effective implementation. The absence of prosecutions of sexual violence against women is indicative of such a dynamic. This outcome is the product of a number of factors. First, such crimes are rarely reported to law enforcement officials for fear of retaliation, rejection, ostracism, or reputational damage (Aroussi, 2011; tinyurl.com/pe4b7t2). Secondly, individuals are often unwilling to come forward because they have little faith that anything will be done (Arab Human Development Report, 2009; tinyurl.com/ntggu3). Support for this second factor includes a report by UN Women (2013; tinyurl.com/oxshu8h), which found that $93.4 \%$ of sexual harassment victims in Egypt did not report incidents to law enforcement officials. According to Ilahi 


\section{HarassMap: Using Crowdsourced Data to Map Sexual Harassment in Egypt}

Chelsea Young

(2009; tinyurl.com/7rxu6hf), a major contributor to this dynamic is that police officers "are some of the worst harassers and therefore, it becomes pointless to seek help from them". This perception of the police also suggests the number of incidents of sexual harassment that are reported through formal channels may severely understate the magnitude of the problem.

Through the use of a crowdsourcing model, HarassMap is able to gather data on a sensitive issue that, despite being widespread, has received relatively little attention from formal law enforcement officials. The HarassMap example highlights a significant opportunity for further applications of this technology in environments characterized by strong organizational cultures or poor governance structures, where detection of specific issues may be similarly hindered by cultural or institutional constraints.

\section{Community-level benefits}

By publically documenting instances of sexual harassment, HarassMap helps to raise awareness among community members and challenge social misconceptions regarding sexual harassment. In Egypt, deeply embedded notions of gender inequality have perpetuated a view of women as a source of "social chaos" that has a corrupting influence on men (Ilahi, 2009; tinyurl.com/ 7rxu6hf). Not only do such notions imply that victims are responsible for the sexual harassment they incur, but they feed cultural practices that favour female purity, place women in a subordinate status to men, and make women vulnerable to forms of gender-based violence (Bowman et al., 2008; tinyurl.com/lndumc3). These perceptions have resulted in a number of formal laws and cultural practices that restrict women's access to the public sphere and effectively institutionalize sexual harassment (Arab Human Development Report, 2009; tinyurl.com/ntggu3). By documenting and reporting instances of sexual harassment, HarassMap helps to challenge the social acceptability of such behaviour and underscore the responsibility of community members to intervene to stop harassment. This data is then used to support HarassMap's offline community-mobilization campaigns, which seek to break down misconceptions that sexual harassment is limited to certain neighbourhoods or that it is only a problem facing women who wear revealing clothing. HarassMap's volunteers play a key role in accomplishing these aims by taking to the street to engage with community members to establish "safe areas" across the city where victims of harassment can find refuge. These community members are provided with training from HarassMap staff on the appropriate protocol when intervening to stop harassment. Currently, these campaigns operate on a monthly basis in 21 communities and 17 governorates in Egypt (tinyurl.com/klvl8bc). HarassMap also reports that approximately $80 \%$ of the community actors approached as a result of these initiatives have become involved in making their community a "zero-tolerance zone for harassment" (tinyurl.com/klvl8bc). Through these efforts, HarassMap challenges the prevailing discourse surrounding sexual harassment in Egypt and opens up a space for the development of informal support systems for women where victims can openly share their experiences in a safe environment.

Community-level lessons can also be drawn from the accessibility of HarassMap's model. By overlaying instances of harassment on a map with a marker that corresponds to the number of reports originating in these areas, HarassMap overcomes language and cultural barriers to communication, thereby allowing the message to be widely understood and dispersed (PSFK, 2011; tinyurl.com/6xcsag4). The visual representation of crowdsourced data has a positive impact on the usability and consumption of data among stakeholders, thereby improving the effectiveness of HarassMap's offline programming.

\section{National-level benefits}

At the national level, the information gathered by HarassMap may prove useful in facilitating prompt and informed decision making. The use of a crowdsourcing model permits HarassMap to quickly make available information that can be used to monitor the well-being of high-risk communities without requiring significant investments in human resources or infrastructure. Such a technology has tremendous potential for situations in which rapid response is a critical success factor (PSFK, 2011; tinyurl.com/6xcsag4). Crowdsourcing technology reduces lead-time, thereby allowing the data gathered on the platform to improve the efficiency and effectiveness of active mitigation strategies. For instance, by highlighting areas with frequent instances of sexual harassment, HarassMap can help law enforcement officials to allocate personnel more efficiently or to identify and arrest specific offenders. For examples of user reports, see the HarassMap interactive map: harassmap.org/en/what-wedo/the-map/

\section{Global-level benefits}

The success of HarassMap in Egypt has drawn attention to the issue of sexual harassment by international organizations engaged in development work, and has led 


\section{HarassMap: Using Crowdsourced Data to Map Sexual Harassment in Egypt}

Chelsea Young

to the development of similar initiatives in eight other countries (tinyurl.com/nm9ngm9). The spillover of this initiative into other locales is partially attributable to the adaptability and accessibility of the model. The prevalence of mobile technology in the developing world - in 2010 , over $80 \%$ of the world's mobile devices were located in developing countries (United Nations Global Pulse, 2012; tinyurl.com/morpmnd) - has enabled HarassMap to overcome traditional issues of access and engage citizens to begin to address the collective issue of sexual harassment on a global level.

Given that mobile devices enable citizens in many different contexts to easily contribute to the initiative without significantly changing their routines, HarassMap's model may hold strong potential to enhance research on international development issues and transform the manner in which stakeholders engage in data collection. Crowdsourcing platforms represent an opportunity for researchers to overcome common barriers to data collection and gather "richer, deeper insights into human experience" (United Nations Global Pulse, 2012; tinyurl.com/morpmnd). For instance, by facilitating the rapid collection and organization of data, crowdsourcing can help researchers to provide analysis on current issues, thereby mitigating the risks related to the relevance of data. In contrast, traditional research methods carry a considerable time delay, which decreases the relevance of the findings as results become outdated. Crowdsourcing data may similarly prove valuable in mitigating environmental risks and ethical research issues. In areas prone to violence or in remote communities where issues of access may prove challenging for traditional data collection methods, such as surveys, crowdsourcing data can help to ensure the personal security of the researcher and the research subject. It is also possible that the anonymity afforded by such a model may prompt more individuals to come forward to share their experiences, thereby improving both the quantity and quality of the data generated from such platforms.

\section{Reality and Potential Limitations}

Despite HarassMap's noted success in raising awareness among community members and challenging prevailing norms regarding sexual harassment, there are nevertheless a number of limitations to this technology that may pose a challenge to accomplishing the project's goals.

The issue of security is particularly crucial given the problem that HarassMap seeks to address. For ex- ample, a common reality of such platforms is that the owner of the platform has little control over how the data will be used once it has been uploaded and published. Lloyd (2011; tinyurl.com/ornbt9y) draws attention to the risk that individuals who choose to share online reports on events relating to human rights abuses could be placed in significant danger should "oppressive officials" elect to identify the source of the data. This scenario draws attention to the need for platform owners to take precautionary measures to ensure the security of contributors. Examples of such measures may include protecting the anonymity of contributors and taking steps to anticipate how this data will be used once it has been published. (Lloyd, 2011; tinyurl.com/ornbt9y).

Indeed, HarassMap has implemented a number of precautionary measures aimed at minimizing such security risks. The most basic of these provisions is the anonymity afforded to the contributor. Upon filing a report, contributors are given the opportunity to provide a name and an email address (which remains confidential and is not published to the website), or they may elect to remain anonymous. These contributors are also asked to voluntarily attach a news report to their submission, if it is available. After the report has been submitted, it is examined by one of HarassMap's volunteers, who verifies the attached information and ensures that personal details, such as the name of the harasser, are omitted from the report before uploading it to the HarassMap website. Where corresponding evidence, such as a news report, has not been provided, a volunteer examines the report and, if it is determined to be reasonable, it is uploaded to the HarassMap website and flagged as an "unverified" report. This control relies on the value judgments of volunteers that have been trained to detect false reports. However, because this control process is not foolproof, there nevertheless remains a risk that the reports published by HarassMap may be inaccurate or false, which poses a number of risks for the project. First, a high incidence of unreasonable or inaccurate reports could undermine the integrity of the project and pose a serious risk to its sustainability. Second, false reports could be used by individuals to target specific neighbourhoods or demographic groups and, in extreme cases, may even provoke retaliatory violence. Given the potential impact of these risks, it is crucial that platform owners take responsibility for considering the potential uses of data and adopt measures to ensure that such risks are minimized.

Although such measures indicate a strong intent to provide assurance on the veracity of the data before it is 


\section{HarassMap: Using Crowdsourced Data to Map Sexual Harassment in Egypt}

Chelsea Young

published to the HarassMap website, the reality of the crowdsourcing model is that there is little that can be done to conclusively demonstrate the accuracy of large quantities of "good-enough" data collected in real time (Lloyd, 2011; tinyurl.com/ornbt9y). Goldman (2012; tinyurl .com/bwzn5lv) discusses these challenges in the context of a similar platform, Crisis Tracker, which mines crowdsourced data coming out of Twitter so that journalists and policy makers can rapidly make use of this information in crisis situations. Given the potential use of such data as evidentiary support in policy formulation, Goldman explores whether crowdsourcing platforms have an ethical or legal obligation to verify information before publishing it. Although Goldman (2012; tinyurl .com/bwzn5lv) acknowledges the shortcomings and risks associated with the use of this data, she concludes that to verify this information would require significant resources in the form of personnel and time, and that the appropriation of these resources may negate the benefits afforded by such a platform to begin with. In such a context, the organization in question must assess the risks and its own risk tolerance when deciding to publish this data.

Fruchterman (2011; tinyurl.com/pg7daqr) highlights an additional weakness of crowdsourcing data by noting that, where SMS technology is used to measure public violence, it is possible that the only reports registered will be those that have not been suppressed by aggressors. As such, the information gathered may reveal a false positive. He explains that it is likely that little information could be expected to originate in areas where technology or, more grimly, victims have been suppressed. As a result, such findings may provide the false indication that no violence has occurred in these areas, when in fact these areas are hotspots of violence. In the case of HarassMap, this scenario may by applicable to regions where there is a high level of stigmatization and shame facing victims of sexual harassment. Under such circumstances, this may produce an absence of reports from a geographic area that is, in actual fact, highly prone to such attacks.

There is an additional risk that the data gathered as a result of crowdsourcing may not form a representative sample. The assumption is that individuals with knowledge of HarassMap and access to mobile devices or computing technology may represent only a subset of the population. As such, the data gathered via HarassMap may not be indicative of the level of sexual harassment taking place in the community. This risk poses a serious challenge to the usefulness of the data gathered as a result of this platform. However, the increased availability of mobile devices in the developing world would seem to challenge any direct issues of access. What is more likely is that informal institutions and cultural constraints operating in these contexts may pose a greater barrier to the collection of representative data.

Although crowdsourcing data raises a number of concerns regarding integrity and quality of data, especially where such data is used to support policy formulation, some proponents have argued that the quality of this data may be "good enough" given its tremendous potential to facilitate the development of more responsive policy and to rapidly inform decision making in changing situations (United Nations Global Pulse, 2012; tinyurl.com/morpmnd). In this case, where the issue in question is highly taboo, the crowdsourcing model proves to be particularly adept in collecting hard-togather data.

\section{Future Implications}

Recently, social media platforms have been celebrated for their predictive potential, particularly those in the health sector. In Haiti, information coming out of Twitter accurately predicted a Cholera outbreak before officials had begun to understand what was going on (Hirschfeld, 2012; tinyurl.com/onmphev). In the case of HarassMap, it may be possible to prevent instances of violence against women using social media monitoring to identify high-risk areas through the flow of information, thereby allowing stakeholders to rapidly mobilize and target their resources most effectively.

A further extension of HarassMap may involve circumventing the need for individuals to upload their content directly to the platform. In its current state, HarassMap is dependent upon victims or witnesses having prior knowledge of the project and access to mobile devices in order to report sexual harassment. In the future, it may be possible to reduce issues of access by aggregating data coming out of multiple social media platforms, thereby enabling the mapping of sexual harassment without requiring participants to have prior knowledge of the HarassMap platform. However, given the vast amount of data available on such platforms, this possibility raises additional challenges such as organizing and making sense of this data and merging it on a common platform. It also raises additional ethical concerns related to privacy and ownership of information. 


\section{HarassMap: Using Crowdsourced Data to Map Sexual Harassment in Egypt}

\section{Chelsea Young}

\section{Lessons Learned}

This article has focused on the application of crowdsourcing technology to address the issue of sexual harassment in Egypt. However, many of the lessons emerging from this case study hold relevance beyond the field of social policy:

\section{For organizations}

The application of crowdsourcing technology in business has been celebrated for enabling greater access to internal expertise and knowledge (Guillot, 2013; tinyurl.com/kcfztn7). However, this technology may prove equally as valuable in enterprise risk management. For instance, crowdsourcing technology could be used as a tool to facilitate a holistic view of risk within an organization. By accessing "the wisdom of the crowd", crowdsourcing can be applied to raise awareness about risks that, on their own, may be triaged as low-priority but when aggregated reveal a very different story (Guillot, 2013; tinyurl.com/kcfztn7). As the case of HarassMap illustrates, the detection of specific issues or risks can be seriously impeded by cultural or institutional factors. In this sense, the application of crowdsourcing technology may prove valuable in navigating cultural and governance constraints to effective oversight.

Applied to specific problems, crowdsourcing technology can be used by businesses to improve the efficiency and effectiveness of mitigation strategies by facilitating rapid and informed decision making based on "good-enough" data. Similarly, crowdsourcing technology may present an innovative means for organizations to efficiently monitor the performance of high-risk projects.

\section{For researchers}

Perhaps the most notable advantage of crowdsourcing technology is the reduced lead-time associated with data collection. This factor carries enormous benefits for researchers. Crowdsourcing data enables researchers to provide insight into current issues, thereby reducing the risk associated with the relevance of findings. Where the issue in question is highly taboo, as is the case with sexual harassment in Egypt, the crowdsourcing model proves to be particularly adept at navigating issues of access and collecting hard-to-gather data. HarassMap illustrates how the use of mobile technology can further reduce these barriers to access and make it easier for actors to participate in research studies. This approach not only further reduces the leadtime associated with data collection, but it may also have a positive impact on the quality and the quantity of the data collected because this methodology minimizes the disruptive impact of the research on the participants.

Crowdsourcing as a research methodology may also be highly attractive from the standpoint of researchers engaged in data collection in remote or high-risk areas where traditional research methods may place the researcher in considerable risk. In such a context, crowdsourcing can be applied to reduce risks related to the personal security of the researcher and also the research subjects.

However, this case study has also highlighted a number of shortcomings related to the integrity of crowdsourced data, which may pose challenges for the application of this data in research studies. In particular, verifying the accuracy of crowdsourced data continues to be a concern that raises a number of ethical issues related to broader applications of this data. Despite attempts to screen this data for "reasonableness", as is the case with HarassMap, there nevertheless remains a risk that inaccurate information may be presented as fact and that the data may reveal misleading trends. IDRC is currently supporting HarassMap in investigating the usefulness of crowdsourcing as a research methodology.

\section{For platform owners/entrepreneurs}

The potential uses of crowdsourced data raise several concerns and potential risks for platform owners. Through a discussion of the potential consequences arising from the issue of the integrity of crowdsourced data, this case study has drawn attention to a number of ethical issues for actors engaged in collecting or disseminating crowdsourced data. Perhaps the most critical of these concerns is the issue of security. The case of HarassMap has underscored the responsibility of platform owners to ensure that this crowdsourced information does not cause undue harm to others. Platform owners must take responsibility for ensuring that unintended consequences are minimized. This difficult task may be made easier by implementing a number of controls, including screening reports for harmful content, flagging reports as unverified, and giving thoughtful consideration to the multitude of ways in which this information could be applied.

This case has also underscored the value in effectively communicating data and the impact that this can have on increasing the accessibility and consumption of in- 


\section{HarassMap: Using Crowdsourced Data to Map Sexual Harassment in Egypt}

\section{Chelsea Young}

formation. The manner in which HarassMap elected to communicate data had a profound impact on the usability of this information. The map itself serves as a helpful, high-impact visual aid for volunteers when they engage with community members regarding the problem of sexual harassment in their neighbourhood. This method of communicating data permits HarassMap to overcome language and cultural barriers to communication, allowing the core message of the initiative to be understood by a wide audience.

The question of sustainability is of critical concern to platform owners seeking to use crowdsourcing technology to address social problems. As in the case of HarassMap, the sustainability of these platforms as social enterprises may depend heavily on the benevolence of governments or multinational human rights organizations. Although HarassMap was able to survive for two years as an entirely volunteer-run initiative, it was able to expand the scope of its programming and thrive with external funding. Continued external partnerships that provide resources and support may be paramount to the project's survival, especially given that HarassMap continues to be a project and not a legal entity.

Platform owners that are not engaged in addressing social problems must nevertheless address the question of "who pays" for the costs associated with administering such platforms. This task may require platform owners in other domains to adopt innovative business models in order to generate revenue from an aspect of the platform.

\section{Conclusion}

Through the application of crowdsourcing technology to address the issue of sexual harassment, the case of HarassMap has revealed a number of unexpected lessons for researchers and practitioners. Whether this technology will prove successful in driving further innovation in this sector will depend largely on the risk tolerance of organizations in weighing the potential, the challenges and limitations, and the future opportunities of this emerging technology.

\section{About the Author}

Chelsea Young is a research award recipient at the International Development Research Centre (IDRC) in Ottawa, Canada. She is currently working with the Risk Management and Internal Audit department, and her research is focused on the role of internal audit in strengthening accountability and effectiveness in public financial management in developing countries. Previously, she supported the consulting function of Frame, a research oriented NGO based in Beirut that offers multidisciplinary services to advance initiatives on good governance, citizen participation, conflict and peace, and strategic development. She holds an MBA in International Development Management from Carleton University's Sprott School of Business in Ottawa, Canada, and a BA in Political Science and History from the University of Ottawa.

Citation: Young, C. 2014. HarassMap: Using

Crowdsourced Data to Map Sexual Harassment in

Egypt. Technology Innovation Management Review. March 2014: 7-13.

Keywords: crowdsourcing, HarassMap, user contribution systems, international development, social policy, data collection 